Erfahrungen aus der Gutachtertätigkeit

\section{Positive Bilanz}

\section{Ein wesentlicher Bestandteil der EG-Öko-Audit-Verordnung ist die Installation eines Umweltgutachters zwischen Wirtschaft und Öffentlichkeit. Dieser hat eine sehr schwierige Aufgabe zu erfüllen, die aber angesichts der Rahmen- bedingungen in der Regel gut gemeistert wurde.}

$\mathrm{D}$ Von Joachim Ganse er Umweltgutachter prüft auf der Basis der Verordnung standortbezogen die Umweltpolitik und -programme, Managementsysteme, Prüfungs- und Betriebsprüfungsverfahren sowie Umwelterklärungen und erklärt letz-tere für gültig. Basis für die Beteiligung am System ist die Einhaltung der gesetzlichen Vorgaben im Umweltschutz für den zu prüfenden Standort. Aus dieser kurzen und knappen Beschreibung der Prüftätigkeiten des Umweltgutachters wird bei genauem Hinsehen sehr schnell klar, welch ein breites Spektrum diese Personen bewältigen müssen. Eine weitere Prämisse ist, daß diese Tätigkeit, die standortbezogen durchgeführt wird, in überschaubarer Zeit und mit hoher Prüfqualität durchzuführen ist. Wohl wenigen Beteiligten war klar, daß die Schaffung des neuen Berufes Umweltgutachter quasi per Dekret nicht über Nacht erfolgen kann. Daß wir mit dieser Prüftätigkeit im gesetzlich geregelten Raum im Umweltschutz Neuland betreten, hat zur Folge, daß ein Rahmen definiert werden muß, innerhalb dessen sich Unternehmen und Gutachter zu bewegen haben.

Das Öko-Audit-System ist dabei nicht nur Basis einer neuen Unternehmenskultur, sondern auch Basis einer neuen Prüfkultur. Diese Prüfkultur muß sich natürlich erst entwickeln. Dies bedeutet aber auch, daß die gesellschaftlichen Anspruchsgruppen (Öffentlichkeit, Naturschutzverbände, Umweltverbände, Gewerkschaften) einsehen müssen, daß wir es im Bereich des Umweltschutzes mit der Abwendung von traditionellen Werkzeugen des Staates, wie z. B. reiner Grenzwertüberwachung und behördlicher Vollzugsüberwachung, zu tun haben. Längst ist der Staat in diesen Bereichen an seine Grenzen gestoßen. Diente das Ordnungsrecht in den Anfangstagen der Umweltpolitik, der Gefahrenabwehr, zeigte sich in der Folgezeit seine beschränkte Anwendbarkeit für die Belange des Umweltschutzes. Die Wirtschaft - insbesondere die kleinen und mittleren Unternehmen - aber auch die Vollzugsbehörden können heute die Flut immer neuer Gesetze und Verordnungen kaum noch bewältigen.

Die neuen Instrumente der Umweltpolitik und die Schwerpunktverlagerung zu innovativen Lösungen im Sinne einer neuen Aufgabenverteilung von staatlicher Rahmensetzung und unternehmerischen eigenverantwortlichem Handeln werden nun begonnen. Entscheidungsträger der Unternehmen haben künftig ökologische Unternehmensziele zu formulieren und deren Erfiillung durch ein Öko-Controlling-System zu planen, zu lenken und zu kontrollieren. Mit dem neuen Instrument „Umweltmanagement-Umwelt-AuditSystem " gilt der Fokus einer modernen Unternehmensführung. Proaktives Umweltmanagement heißt dabei, die aus der betrieblichen Tätigkeit resultierenden Umweltauswirkungen zu planen, zu steuern und zu kontrollieren.

Betrachtet werden dabei sämtliche Aspekte, Verfahren, Abläufe, Verantwortlichkeiten und Mittel. Ein Umweltmanagement umfaßt dann alle Methoden und Verfahren, die für die Aufgabe eingesetzt werden, mit dem Ziel der fortlaufenden Verbesserung der Umweltleistung eines Unternehmens. Diese unternehmensinternen Kontrollfunktionen geben der Unternehmensleitung Informationen über die Effizienz der Umweltmanagementsysteme und das vorhandene Controlling-System.

\section{Anforderungen an die Gutachter}

Für den Umweltgutachter bedeutet das, daß er bei seiner Prüftätigkeit auf einer höheren Abstraktionsebene, nämlich im Managementsystem, ansetzen muß. Darüber hinaus bedeutet es, daß er die Einhaltung aller Vorschriften dieser Verordnung, insbesondere in Bezug auf

- die Umweltpolitik und das Umweltprogramm, die Umweltprüfung, das funktionierende Instrumentensystem, das Umweltbetriebsprïfungsverfahren und die Umwelterklärung, die Zuverlässigkeit der Daten und Informationen der Umwelterklärungen und

- die ausreichende Durchführung aller für den Standort relevanten Fragestellungen in dieser Erklärung

zu prüfen hat. Dies heißt, daß er indirekt über die vorliegende Umweltprüfung prüft, ob die Anforderungen erfüllt sind (ComplianceCheck). Darüber hinaus hat er sich zu vergewissern, daß das Unternehmen ein risikoadäquates Umweltmanagementsystem aufgebaut hat.

Dies hat mehrere Implikationen. Die Gutachter greifen im wesentlichen auf die vorliegenden Grundlagen aus der Umweltprüfung und den Umweltbetriebsprüfungen zurück, vergleichen aber auch im Umwelthandbuch niedergelegte Abläufe mit dem vorgefundenen Zuständen (Inaugenscheinnahme). Es soll dabei im wesentlichen der Charakter einer Systemprüfung erhalten bleiben, jedoch wird der Umweltgutachter das Funktionieren der Umweltmanagementsysteme (Not-/Krisenfall, Produktgestaltung) auf den Hauptwirkungspfaden des Unternehmens zu begutachten haben.

Das Unternehmen muß ein risikoadäquates Managementsystem entwickelt haben. Risikoadäquat bedeutet, daß die Umweltauswirkungen der Tätigkeiten am Standort bekannt und entsprechende Systeme vorhanden sein müssen, um Wasser, Boden und Luft zu schützen.

Das damit dem Umweltgutachter eine sehr kreative Rolle zufällt, war bei der Verabschiedung der Verordnung mit Sicherheit nicht so bewußt: In der Regel wird ein Gutachter der Unternehmensleitung aufgrund seiner Prüftätigkeit strategische Hinweise aus der „Vogelperspektive“ zur weiteren Entwicklung des Managementsystems geben können und müssen.

Folglich sind die Anforderungen an den Umweltgutachter fachlich, berufsrechtlich und persönlich sehr hoch. Einige Anforderungen möchte ich hier kurz nennen, $z$. B. Unabhängigkeit, Verschwiegenheit, Unparteilichkeit, aber auch das Abstraktionsvermögen, Kommunikationsfähigkeit und die Ausgewogenheit in der Bewertung der vorgefundenen Unternehmenssituationen.

Die bisherige Prüftätigkeit der DAU (Deutsche Akkreditierungs- und Zulassungsgesellschaft für Umweltgutachter) hat bewiesen, daß wir in Deutschland an guten Grundstock für derart qualifizierte Personen haben. In einem recht harten Auswahlverfahren hat sie in Deutschland 
bisher ca. 200 Einzelgutachter zugelassen. Über 1200 Standorte sind in Deutschland durch Gutachter bzw. Gutachterorganisationen geprüft worden.

\section{- Positive Zwischenbilanz}

Das System hat die erste Bewährungsprobe meines Erachtens bestanden, steht aber nun vor dem nächsten Evolutionsschritt. Dies bedeutet natürlich, daß der Erfahrungsaustausch zwischen den Umweltgutachtern viel stärker forciert werden muß. Hier ist seitens der beteiligten Parteien Bundesumweltministerium, Umweltgutachterausschuß und dem Institut der Umweltgutachter und -berater in Deutschland (IdU) e. V. Handlungsbedarf in der Aus- und Weiterbildung der Gutachter erkannt worden. Die Erfahrung, die bisher in den Validierungsverfahren gesammelt wurde, muß zur Anhebung und Angleichung des Qualitätsniveaus herangezogen werden. Gewisse Rahmengegebenheiten wie Prüftiefe, Prüfumfang und Mindestprüfzeiten sollten im Rahmen eines institutionalisierten Erfahrungsaustausches festgelegt werden.

Hierzu ist es aber erforderlich, daß das Selbstverwaltungssystem der Umweltgutachter (berufsständische Vertretungen wie das IdU) seitens der beteiligten Kreise stärker gestützt wird, um somit dem Umweltgutachter die ihm zugedachte Position als unabhängiger Garant der Fortentwicklung des EMAS-Systems und der Umweltleistung der Unternehmen zu ermöglichen. Nur so können wir einen modernen, kostengünstigen, innovativen Entwicklungsprozeß in Produktionsverfahren sowie Dienstleistungsprozessen erreichen und die Akzeptanz von EMAS in der Wirtschaft erhöhen. Der Grundstein ist gelegt - der weitere Aufbau hat im Sinne der ökologischen und ökonomischen Entwicklung der Unternehmen unverzüglich und praxisnah $z u$ erfolgen.

\section{Der Autor}

Joachim Ganse ist Geschäftsführer von Gerling Cert. und Vorsitzender des Instituts der Umweltgutachter und -berater in Deutschland (IdU) e.V.

Kontakt: Gerling Cert., Frankfurter Str. 720-726, 51145 Köln. Tel. 0221/144-50 12, Fax -76 66
Die Stellung des Umweltgutachters im Licht der bisherigen Erfahrungen

\section{Problematische Rolle}

\section{EMAS war ursprünglich als reine Audit-Verordnung angelegt: Die Unternehmen sollten durch eine unabhängige Überpröfung ("audit") motiviert werden, ihre Umweltverantwortung wahrzunehmen, und in die Lage versetzt werden, ihre Umweltaktivitäten der interessierten Öffentlichkeit gegenüber glaubhaft dar- zulegen. Hierfür sollte nicht zuletzt der Umweltgutachter sorgen. Um seine Funktion als Garant der Glaubwürdigkeit und Funktionsfähigkeit des Systems sicherzustellen, muß eine fachkundige, unabhängige und neutrale Aufgaben- wahrnehmung gewährleistet werden.}

$\mathrm{D}$ Von Ludwig Glatzner ie Idee, durch ein glaubwürdiges Informations- und Prüfungsverfahren den Unternehmen Vertrauen, Ansehen und Akzeptanz in der Öffentlichkeit zu verschaffen, war und ist charakteristisch für EMAS. Allerdings wurde sehr schnell erkannt, daß eine Prüfung bestimmter Vorgaben bedarf, die dann auch in Form von Anforderungen an ein ,Umweltmanagementsystem“ formuliert wurden. Der Umweltgutachter, der sowohl die vom Unternehmen in Form der Umwelterklärung bereitgestellten Informationen zu überprüfen hat (,Validierung") als auch das Unternehmen selbst auf Einhaltung aller Vorgaben begutachtet (,Verifizierung"), nimmt dabei für das ganze System, insbesondere für dessen Glaubwürdigkeit und Funktionsfähigkeit, eine zentrale Rolle ein. Er soll nicht nur dafür sorgen, daß die Konformität der Unternehmensrealität mit den Vorgaben direkt festgestellt wird; von der Arbeit des Umweltgutachters hängt auch das Funktionieren weiterer System-Elemente $\mathrm{ab}$, wie insbesondere die „Eigenkontrolle“ durch das interne Audit, die „Endkontrolle“ im Rahmen der Registrierung oder auch die „Fremdkontrolle“ des Unternehmens beispielsweise durch interessierte Kreise oder Institutionen der Öffentlichkeit. Erfahrungen mit dem Umweltgutachter werden im folgenden insbesondere vor dem Hintergrund des Systemvergleichs EMAS - ISO 14001, der (deutschen) Zulassungs- und Aufsichtspraxis und der Qualität der Aufgabenerledigung durch die Umweltgutachter diskutiert.

Das deutsche Umweltgutachter-Modell kann als ,individual approach“ charakterisiert werden. Unabhängig davon, ob Einzelpersonen oder Organisationen in Erscheinung treten, beruht die Tätigkeit als Umweltgutachter immer auf der
Basis einer individuellen, personengebundenen Zulassung, Prüfungsarbeit und Verantwortlichkeit. Die „ISO-Welt“ dagegen kennt nur Zertifizierungsstellen, also akkreditierte Organisationen, die sich einzelner Auditoren bedienen. Der Zertifizierungsauditor legt der Zertifizierungsstelle seinen Prüfbericht vor, die dann in eigener Verantwortung die Zertifizierungsentscheidung trifft. Diese Trennung von Prüfung und Entscheidung bei der Zertifizierung wird zum Teil als Gegencheck zur Qualitätssicherung interpretiert, zum Teil jedoch auch als Einfallstor prüfungsferner Entscheidungskriterien kritisiert. Der Umweltgutachter hingegen wird immer selbst vor Ort tätig und verantwortet auch seine Prïfungsentscheidung selbst (1). Die Qualitätssicherung erfolgt extern per Zulassung und Aufsicht durch die DAU GmbH, die staatlich beauftragte Deutsche Akkreditierungs- und Zulassungsgesellschaft für Umweltgutachter.

\section{- Das Zulassungsverfahren}

Im Mittelpunkt der Diskussionen um die Qualität der Zulassung von Umweltgutachtern stand und steht die Fachkundeprüfung. Kritik scheint angebracht zu sein bezüglich der Startphase des Zulassungssystems, in der die Zulassung von Umweltgutachtern vielleicht doch vergleichsweise zu leicht und für zu viele Unternehmensbereiche (,Branchen“) erlangt werden konnte. Ein Beispiel: Man wunderte sich öffentlich in einem Zeitungsartikel darüber, wie denn ein Rechtsanwalt trotz reiner juristischer Tätigkeiten eine Zulassung über alle Branchen erhalten konnte.

Über derartige ,Kinderkrankheiten“ hinaus existiert jedoch auch eine wesentliche Schwachstelle im Prüfungssystem: Der Prüfling kann die Zulassung für eine beliebige Anzahl von Unternehmensbereichen („Branchen“) beantragen, 
(c) 20I0 Authors; licensee IÖW and oekom verlag. This is an article distributed under the terms of the Creative Commons Attribution Non-Commercial No Derivates License (http://creativecommons.org/licenses/by-nc-nd/3.o/), which permits unrestricted use, distribution, and reproduction in any medium, provided the original work is properly cited. 